\title{
Признаки подготовки мощных вспышек на Солнце в сентябре 2017 г. в микроволновом излучении и структуре магнитного поля активной области по данным РАТАН-600 и SDO/HMI
}

\author{
В.Н. Боровик ${ }^{1}$, В.Е. Абрамов-Максимов ${ }^{1}$, А.Г. Тлатов ${ }^{1}$, Л.В. Опейкина ${ }^{2}$, \\ А.Д. Шрамко , Л.В. Яснов ${ }^{3}$ \\ 1 Главная (Пулковская) астрономическая обсерватория РАН, Пулковское шоссе, 65/1, Санкт-Петербург, \\ Россия, 196140 \\ beam@gaoran.ru \\ 2 Специальная астрофизическая обсерватория РАН, Нижний Архыз, Россия, 369167 \\ 3 Санкт-Петербургский государственный университет, Санкт-Петербург, Россия, 198504
}

Поступила в редакцию 14 ноября 2017 г.

\begin{abstract}
Аннотация. Приведены результаты сравнительного анализа микроволнового излучения и магнитографических характеристик AO NOAA 12673 и 12674 с целью выявления признаков подготовки больших вспышек. В AO NOAA 12673 в сентябре 2017 г. произошли самые мощные вспышки 24го цикла солнечной активности (Х9.3 и Х8.2). Развитая АО NOAA 12674, в которой было только несколько слабых вспышек С-класса, использована для сравнения. Анализ основан на данных наблюдений в микроволновом диапазоне на радиотелескопе РАТАН-600 и магнитографичесих наблюдениях на космической обсерватории SDO/HMI. Как и в ранее исследованных случаях, перед первой большой вспышкой в АО NOAA 12673 (M5.5) зарегистрирован резкий рост градиента магнитного поля с последующим его уменьшением непосредственно перед вспышкой и значительное возрастание интенсивности микроволнового излучения АО. В АО NOAA 12674 не обнаружено заметных изменений градиента магнитного поля и микроволнового излучения.

SIGNS OF PREPARATION OF POWERFUL SOLAR FLARES IN SEPTEMBER 2017 IN MICROWAVE RADIATION AND STRUCTURE OF THE MAGNETIC FIELD OF ACTIVE REGION USING RATAN-600 AND SDO/HMI DATA, by V.N. Borovik, V.E. Abramov-Maximov, A.G. Tlatov, L.V. Opeikina, A.D. Shramko, L.V. Yasnov. We present the results of a comparative analysis of microwave radiation and magnetographic characteristics of solar active regions (ARs) NOAA 12673 and 12674 with the purpose of revealing signs of preparation of strong flares. The most powerful flares (X9.3 and X8.2) of the 24th solar cycle occurred in NOAA 12673 in September 2017. The well-developed AR NOAA 12674, in which there were only a few C-class weak flares, is used for comparison. The analysis is based on observational data in the microwave range using the RATAN-600 radio telescope and magnetographic observations at the SDO/HMI space observatory. As in the previously investigated cases, we registered a sharp increase of the magnetic field gradient with its decrease directly before the flare and a significant increase of microwave radiation before the first large flare in NOAA 12673 (M5.5). No significant changes are detected in the gradient of the magnetic field and microwave radiation in NOAA 12674
\end{abstract}

Ключевые слова: Солнце, вспышки, радиоизлучение, прогностический фактор, магнитное поле 


\section{1 Введение}

Попытки выявить предвестники больших вспышек на Солнце по изменениям структуры магнитного поля активных областей $(\mathrm{AO})$ и микроволновому излучению предпринимаются уже несколько десятилетий.

Первые указания на связь вспышечной активности со структурой магнитного поля АО были получены более 50 лет назад. Кюнцель (1960) пришел к выводу, что образование $\delta$-конфигурации в АО является наиболее значимым предвестником высокой вспышечной активности АО. Северный (1960) выявил тесную связь возникновения вспышек с особенностями структуры магнитного поля АО. В ряде работ последних лет (Шривер, 2016; Харра и др., 2016; Ториуми и др., 2017) сделан вывод, что наиболее характерным признаком подготовки большой вспышки является образование структуры магнитного поля типа “SHIL” (Strong-field, High-gradient polarity Inversion Line).

Первые свидетельства существования микроволновых источников, проектирующихся на межпятенную область, и их связи со вспышками были получены Пулковской группой радиоизлучения Солнца по наблюдениям солнечного затмения 7 марта 1970 г. (Киненес и др., 1975). Наблюдения Солнца на WSRT на волне 6 см в 1974 г. и последующие наблюдения на этом радиотелескопе обнаружили квазистационарные яркие источники над нейтральной линией во вспышечно-активных областях (Кунду и др., 1977; Кунду, Алиссандракис, 1984).

Начавшиеся в 1975 г. регулярные наблюдения Солнца в микроволновом диапазоне на РАТАН-600 выявили “пекулярные” радиоисточники в АО перед большими вспышками над местами максимального градиента фотосферного магнитного поля вблизи нейтральной линии, которые часто становились доминирующей компонентой в микроволновом излучении всей АО (Ахмедов и др., 1986).

Ежедневные наблюдения на радиогелиографе Нобеяма (NoRH) на волне 1.76 см показали, что источники над нейтральной линией магнитного поля (neutral line associated source - NLS) - типичные и часто доминирующие источники в АО, в которых происходят большие вспышки. Была найдена связь между появлением NLS и началом вспышки (Уралов и др., 2008). Рождение и развитие этих источников связано с возникновением больших вспышек и может рассматриваться как прогностический фактор.

Много результатов получено в последние годы благодаря новым наблюдательным возможностям, в частности, магнитографическим наблюдениям на космической обсерватории SDO и наблюдениям в микроволновом диапазоне на радиотелескопе РАТАН-600 с использованием многооктавного спектрально-поляризационного комплекса высокого разрешения (Богод и др., 2011).

По наблюдениям на РАТАН-600 было показано, что в исследованных АО, наблюдавшихся в 20112015 гг., за 1-2 дня до вспышек класса X (по классификации GOES) в микроволновом излучении АО регистрировался новый (в большинстве случаев доминирующий) микроволновый источник (NLS), который ассоциировался с местом максимального градиента фотосферного магнитного поля вблизи нейтральной линии (Абрамов-Максимов и др., 2015а). Эволюция этого источника была подобна эволюции градиента магнитного поля (Абрамов-Максимов и др., 2015б). Выполненный одновременно магнитографический анализ показал, что в АО регистрируется быстрый рост горизонтального градиента магнитного поля, вспышка происходит через 5-20 часов после максимума градиента.

Цель данной работы - продолжить цикл работ авторов по выявлению признаков подготовки больших вспышек (классов М5-X по рентгеновской классификации) в АО Солнца по микроволновому излучению и по магнитографическим характеристикам АО. В данной работе приведены предварительные результаты анализа AO NOAA 12673, в которой в сентябре 2017 г. произошли самые мощные вспышки 24-го цикла солнечной активности. Для сравнения проанализирована развитая АО NOAA 12674, в которой было только малое количество слабых вспышек С-класса.

\section{2 Наблюдения}

Мы использовали результаты ежедневных спектрально-поляризационных многоволновых наблюдений Солнца, выполненных на радиотелескопе РАТАН-600 в выбранном для анализа диапазоне волн (1.8- 
4.0) см. Наблюдения проводились с помощью многооктавного спектрально-поляризационного комплекса высокого разрешения с регистрацией как интенсивности (параметр Стокса I), так и круговой поляризации (параметр Стокса V) (Богод и др., 2011). Ножевая диаграмма антенны на волне 2.0 см (HPBW) составляет $17^{\prime \prime} \times 13^{\prime}$.

Магнитографический анализ выполнялся по данным SDO/HMI по методике, изложенной в (Тлатов и др., 2014). Вычислялись потоки магнитного поля, локальные горизонтальные градиенты поля в различных частях $\mathrm{AO}$, а также суммарный градиент и его положение.
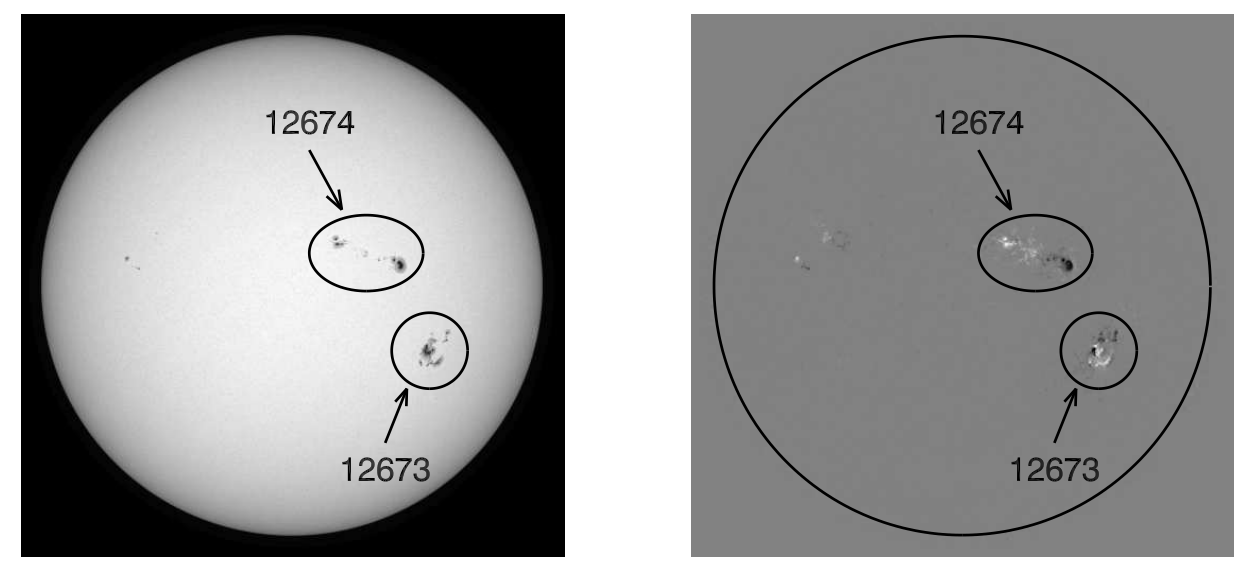

Рис. 1. Изображение Солнца в континууме (слева) и магнитограмма (справа) 6 сентября 2017 г. по данным SDO/HMI. На рисунках указаны AO NOAA 12673 и 12674

На рис. 1 приведены изображение Солнца в континууме и магнитограмма по данным SDO/HMI. Исследуемые AО обведены и указаны стрелками. Обе АО имели примерно одинаковые суммарные площади, которые не превышали 1000 м.д.п. К сожалению, АО 12673 имела компактную структуру, и ее невозможно надежно разделить на отдельные компоненты. Поэтому для анализа микроволнового излучения измерялись интегральные плотности потоков от всей $\mathrm{AO}$.

На рис. 2 и 3 показана детальная структура микроволновых источников, связанных с АО 12673 и 12674. Видно, что в рассмотренный период 3-5 сентября в АО 12674 как магнитная структура, так и структура микроволнового источника остаются стабильными. Спектр микроволнового источника также не претерпевает существенных изменений, что видно на рис. 5. Вспышечная активность АО 12674 низка: в этот период в ней происходит только одна вспышка класса С1.1.

В AO 126733 сентября начинают происходить существенные изменения. Наблюдается всплытие с одновременным вращением магнитного поля и одновременно резкое усиление микроволнового излучения 4 сентября. При этом происходит резкий рост градиента магнитного поля, что видно из рис. 4. Отметим, что первая большая вспышка класса M5.5 произошла 4 сентября в 20:33 UT. Интегральный спектр микроволнового излучения $\mathrm{AO}$ значительно изменяется 3-4 сентября: в несколько раз возрастает интенсивность излучения и увеличивается наклон спектра. Возможно, такое изменение спектра вызвано тем, что к пятенному излучению добавляется излучение развившегося перед вспышкой NLS-источника.

Отметим, что поведение рассмотренной АО 11673 очень похоже на поведение АО 10930, в которой 13 декабря 2006 г. произошла вспышка класса Х3.4: события произошли вблизи минимума солнечной активности, обе $\mathrm{AO}$ имели площадь менее 1000 м.д.п., в обеих АО наблюдалось заметное вращение пятен, в спектрах микроволнового излучения обеих $\mathrm{AO}$ произошли значительные изменения за день до вспышек (Боровик и др., 2007). 

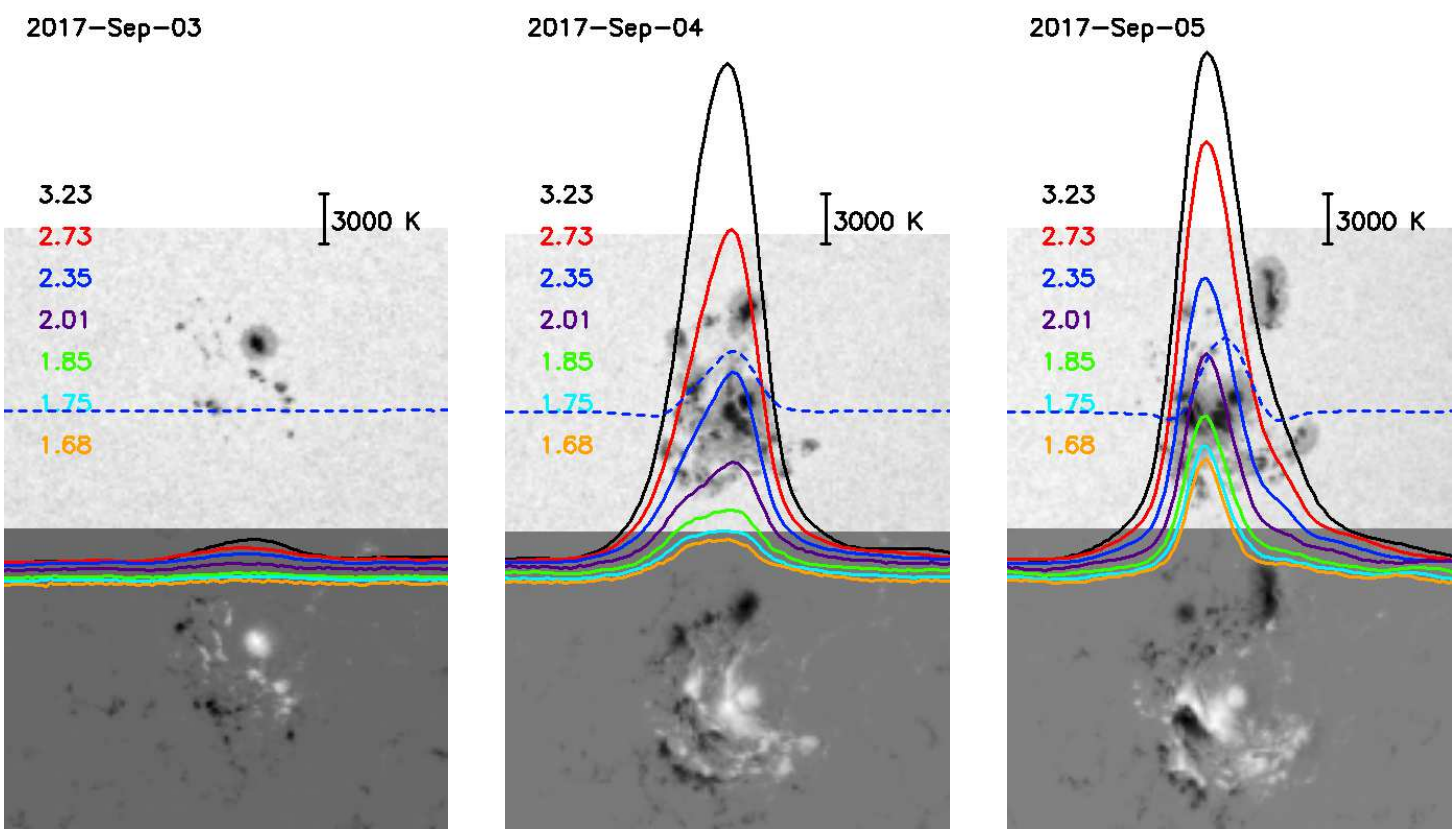

Рис. 2. Фрагменты одномерных сканов Солнца в микроволновом диапазоне, полученные на РАТАН-600 3-5 сентября 2017 г. Сканы наложены на изображения AO NOAA 12673 в континууме и магнитограммы по данным SDO/HMI. Сплошными линиями показаны сканы в каналах интенсивности (параметр Стокса I, длины волн в см даны слева вверху каждой панели), штриховыми линиями показаны сканы в канале поляризации (параметр Стокса V) на волне 2.35 см. Вертикальный отрезок показывает масштаб в антенных температурах (K)

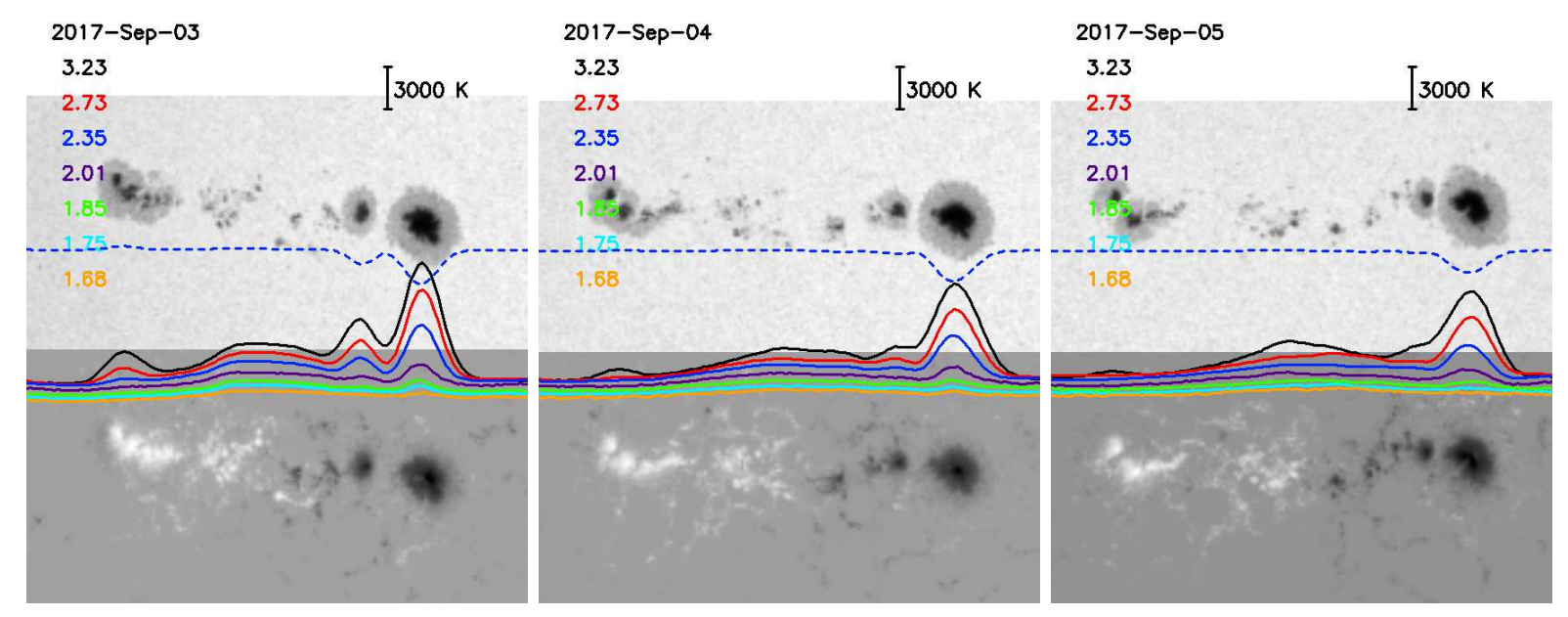

Рис. 3. Фрагменты одномерных сканов Солнца в микроволновом диапазоне, полученные на РАТАН-600 3-5 сентября 2017 г. Сканы наложены на изображения АO NOAA 12674 в континууме и магнитограммы по данным SDO/HMI. Сплошными линиями показаны сканы в каналах интенсивности (параметр Стокса I, длины волн в см даны слева вверху каждой панели), штриховыми линиями показаны сканы в канале поляризации (параметр Стокса V) на волне 2.35 см. Вертикальный отрезок показывает масштаб в антенных температурах (K) 
NOAA 12673

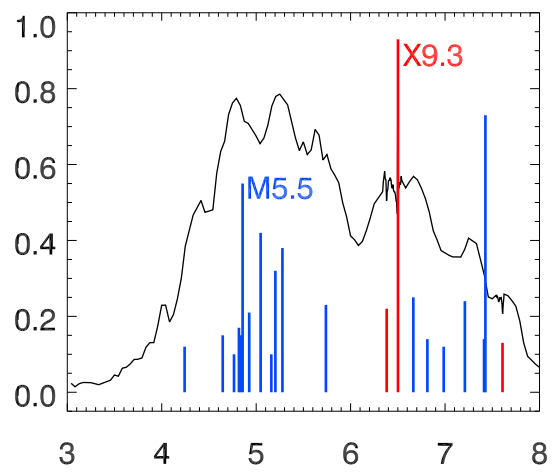

NOAA 12674

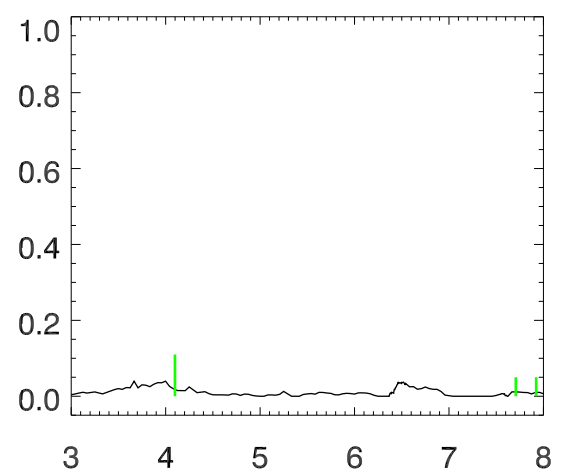

Рис. 4. Эволюция суммарного градиента магнитного поля АО NOAA 12673 (слева) и АO NOAA 12674 (справа). Оси абсцисс - дни сентября 2017 г., оси ординат - градиент, условные единицы. Вертикальные синие линии - моменты М-вспышек, красные - Х-вспышек, зеленые - С-вспышек. Длина линии пропорциональна баллу вспышки
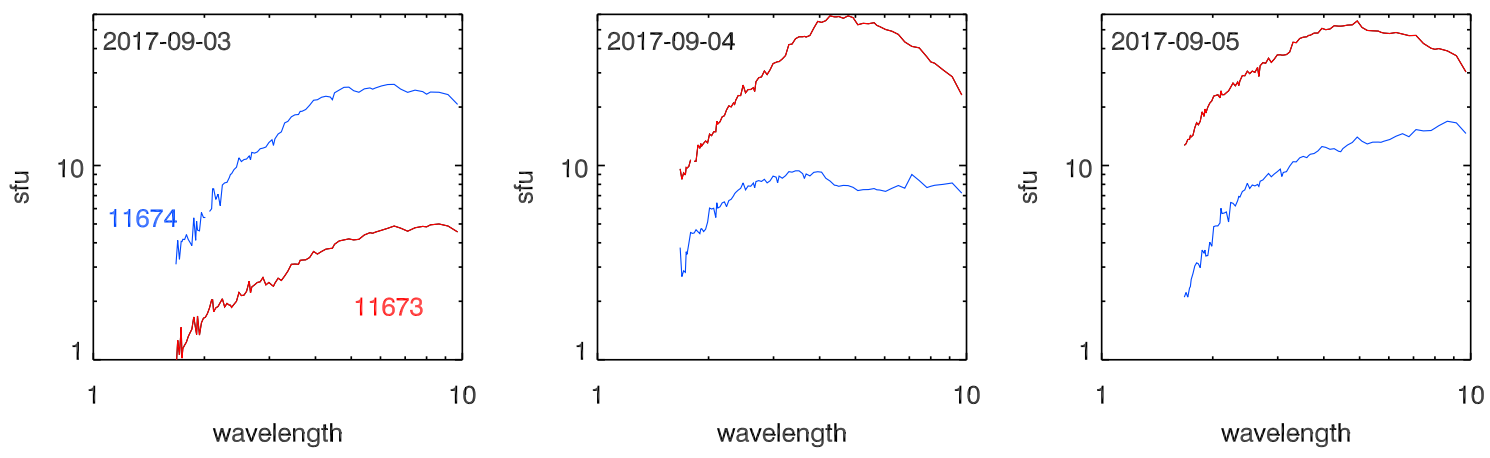

Рис. 5. Интегральные спектры плотностей потоков AO NOAA 12673 (красные кривые) и NOAA 12674 (синие кривые) за 3-5 сентября 2017 г. по наблюдениям на РАТАН-600. Оси абсцисс - длины волн в см, оси ординат потоки в sfu

\section{3 Выводы}

Таким образом, из анализа микроволнового излучения и магнитографических характеристик АО NOAA 12673 и 12674 выявлены следующие наблюдательные факты:

- в исследованной AO NOAA 12673 примерно за день до первой большой вспышки класса M5.5 наблюдается резкий рост градиента магнитного поля с последующим его падением перед вспышкой;

- одновременно с этим происходит увеличение примерно на порядок интенсивности микроволнового излучения $\mathrm{AO}$;

- интегральный спектр микроволнового излучения становится более крутым;

- в AO NOAA 12674, в которой не было заметной вспышечной активности, градиент магнитного поля и микроволновое излучение не испытывают существенных изменений.

Обнаруженные наблюдательные факты находятся в хорошем соответствии с ранее выявленными фактами по результатам исследования событий 2011-2015 гг. Дальнейшее накопление и обобщение таких фактов позволит развить методы прогноза больших вспышек.

Работа частично поддержана Программой Президиума РАН. 


\section{Литература}

Абрамов-Максимов и др. (Abramov-Maximov V.E., Borovik V.N., Opeikina L.V., Tlatov A.G.) // Solar Phys. 2015a. V. 290. P. 53.

Абрамов-Максимов и др. (Abramov-Maximov V.E., Borovik V.N., Opeikina L.V., Tlatov A.G.) // Geomagnetism and Aeronomy. 2015б. V. 55. Issue 8. P. 1097.

Ахмедов и др. (Akhmedov Sh.B., Borovik V.N., Gelfreikh G.B., Bogod V.M., Korzhavin A.N., et al.) // Astrophys. J. 1986. V. 301. P. 460.

Богод В.М., Алесин А.М., Перваков А.А. // Астрофиз. бюллетень. 2011. Т. 66. № 2. С. 223.

Боровик В.Н., Абрамов-Максимов В.Е., Григорьева И.Ю. и др. // ХІ Пулковская международная конференция по физике Солнца "Физическая природа солнечной активности и прогнозирование ее геофизических проявлений”. СПб: Пулково. ГАО РАН. 2007. С. 67.

Киненес Х.А., Коржавин А.Н., Петерова Н.Г., Сантос Х. // Солнечные данные. 1975. № 3. С. 87.

Кунду и др. (Kundu M.R., Alissandrakis C.E., Bregman J.D., Hin A.C.)// Astrophys. J. 1977. V. 213. P. 278.

Кунду, Алиссандракис (Kundu M., Alissandrakis C.) // Solar Phys. 1984. V. 94. P. 249.

Кюнцель (Künzel H.) // Astron. Nachr. 1960. V. 285. P. 271.

Северный А.Б. // Изв. Крымск. Астрофиз. Обсерв. 1960. Т. 22. С. 12.

Тлатов и др. (Tlatov A.G., Vasil'eva V.V., Makarova V.V., Otkidychev P.A.) // Solar Phys. 2014. V. 289. Р. 1403.

Ториуми и др. (Toriumi S., Schrijver C.J., Harra L.K., Hudson H., Nagashima K.) // Astrophys. J. 2017. V. 834. id. 56.

Уралов и др. (Uralov A.M., Grechnev V.V., Rudenko G.V., Rudenko I.G., Nakajima H.) // Solar Phys. 2008. V. 249. P. 315.

Харра и др. (Harra L.K. et al.) // Solar Phys. 2016. V. 291. P. 1761.

Шривер (Schrijver C.J.) // Astrophys. J. 2016. V. 820. id. 103. 